\title{
Juegos lingüísticos y la habilidad comunicativa de los niños y niñas de cinco años de una institución educativa pública de inicial
}

Linguistic games and the communicative ability of five-year-old boys and girls from an initial public educational institution

Mgr. Blanca Mejía Bustillos

Institución Educativa 652 de Supe-Barranca-Perú blamebustillos@outlook.es

\section{Dr. Guillermo Príncipe} Cotillo

Universidad JAIME BAUSATE Y

MEZA

(principegf@hotmail.com)

Recibido

$18 / 08 / 2018$

Revisado $10 / 10 / 2018$

Aceptado $12 / 10 / 2018$

\section{RESUMEN}

El objetivo principal del estudio consistió en demostrar - la influencia de los juegos lingüísticos en el desarrollo - de las habilidades comunicativas de niños y niñas de cinco años de edad de educación inicial. El enfoque adoptado correspondió al cualitativo; tipo de investigación al descriptivo correlacional; y el diseño, no experimental. Se trabajó con una muestra de 60 niños(as). El criterio de selección fue intencional por tratarse de una población pequeña. Durante el proceso de investigación se puso énfasis en el manejo de un conjunto de recursos lúdicos relativos al manejo del lenguaje con el objeto de desarrollar las capacidades con respecto al uso oral y escrito del mismo, partiendo de la distinción de los sonidos inicial y final de las palabras, sumadas al ejercicio divertido y entretenido del músculo de la boca para facilitar la fluidez del habla. De sus resultados se resume que la aplicación sistemática y organizada de los juegos lingüísticos, tiene relación significativa y fuerte con el desarrollo de las habilidades comunicativas oral y escrita de los niños(as), así como con el desarrollo psico-social, con la formación de la personalidad y con la práctica de los valores sociales básicos.

Palabras clave: juegos lingüísticos, habilidades comunicativas, técnicas comprensivas, técnicas expresivas 


\section{ABSTRACT}

The main objective of the study was to demonstrate the influence of language games on the development of communication skills of children of five years of initial education. The approach adopted corresponded to the qualitative one; the type of research, descriptive correlational; and the design, not experimental. We worked with a sample of 60 children (as). The selection criteria was intentional because it was a small population. During the research process, emphasis was placed on the management of a set of recreational resources related to language management in order to develop the capacities with respect to the oral and written use of the same, starting from the distinction of the initial and final sounds of the words, added to the fun and entertaining exercise of the mouth muscle to facilitate speech fluency. From their results it is summarized that the systematic and organized application of linguistic games has a significant and strong relationship with the development of children's oral and written communication skills, as well as with psycho-social development, with training of personality and with the practice of basic social values.

Keywords: linguistic games, communicative skills, comprehensive techniques, expressive techniques. 


\section{INTRODUCCIÓN}

- n el diario quehacer de la actividad lectiva de los docentes de educación - básica regular y, particularmente, de quienes trabajan en el nivel Inicial, uno de los problemas más frecuentes con los que se enfrentan es con la dificultad de desarrollar las competencias comunicativas de niños y niñas de cinco años de edad, ya sea al nivel oral así como en el escrito. Esta dificultad, según se deduce de las disposiciones legales emanadas de las autoridades gubernamentales, debe resolverse con la participación compartida de los dos agentes principales y directos del proceso enseñanza aprendizaje, esto es, los docentes y los alumnos, y teniendo en cuenta la mejor predisposición de ambos elementos para preservar la calidad educativa del país.

Sin embargo, en la praxis educativa diaria, ello no ocurre así. Según experiencia acumulada como docente de aula desde mucho tiempo atrás, en la aplicación de cualquier estrategia didáctica para viabilizar los procesos de enseñanza aprendizaje, se superponen un conjunto de factores administrativos del entorno educativo, los mismos que influyen directa e indirectamente, no solo en el desarrollo de las capacidades comunicativas de los niños y niñas, sino también en la formación integral de los mismos.

Uno de estos factores y quizás el más importante se concentra en torno al diseño e implementación de los currículos de estudio. En efecto, este recurso tal como está concebido y diseñado actualmente resulta ser limitativo, en tanto que se ha elaborado y sigue elaborándose a partir de la visión de los entes administrativos del Gobierno Central, es decir de los organismos concentrados en la capital y, por lo tanto, distante y extraña a las necesidades particulares, propias de los niños y niñas, así como del alumnado en general. Resultas de esta visión unilateral, la formalización del currículo ha devenido en procesos desafortunados, es decir en la implementación de una experiencia ajena a la realidad particular de los estudiantes, particularmente de los infantes de educación inicial.

A tales dificultades se suman otros factores que lindan con lo social, lo económico, lo cultural y acaso también lo estrictamente familiar. En este sentido, en lo que respecta al primero, por ejemplo, se generan problemas de marginación, de exclusión y hasta de racismo; en lo segundo, afectación por el alto costo de la educación, manifestados en el uso limitado de los medios tecnológicos o inaccesibilidad al mismo; y en lo tercero, son proclives a ser afectados por problemas de diversidad cultural, del multilingüismo 
o de la heterogeneidad de costumbres y hábitos; hechos que terminan generando a veces desencuentros, desánimos e inseguridad del alumno.

Esta incongruencia puede constatarse fácilmente y con meridiana claridad al revisar los temas o contenidos insertados en los currículos y los respectivos planes de estudio. Dichos contenidos, en la mayoría de los casos, responden a hechos extraños a la realidad del alumno e inclusive a experiencias ejecutadas en el contexto extranjero, sin la debida evaluación crítica de las bondades que podrían tener para impulsar el aprendizaje de un universo tan sensible, como es el caso de los niños o niñas que se encuentran en pleno proceso de formación.

Otro imponderable que también influye en el desarrollo de la capacidad comunicativa de los niños y niñas, está en relación a la falta de especialización de los docentes en el área de la educación inicial. Por esta falencia se improvisan docentes de aula que, en lugar de viabilizar el cumplimiento de la responsabilidad pedagógica especializada, terminan afectando el mismo proceso formativo de los niños particularmente en lo que respecta al desarrollo de sus capacidades de manejo del lenguaje.

Por esta razón, como una propuesta de solución a aquellos procesos de enseñanza aprendizaje limitativo y hasta coercitivo que se vienen implementando en las instituciones educativas particularmente públicas, en el presente estudio se postula por una didáctica específica. Una didáctica que pondere el manejo de un conjunto de estrategias de carácter lúdico ajustada a las necesidades de desarrollar el manejo fluido, sistemático y racionalizado del lenguaje oral y escrito; con claro supuesto que, a través de dichas actividades, se puede rescatar la capacidad comunicativa de los niños desde la temprana edad, es decir a partir de su conectividad con la realidad o experiencias acumuladas desde el hogar.

Como resultante de esta propuesta se puede afirmar, sin temor a equívoco, que la aplicación de los juegos lingüísticos como estrategia para desarrollar las competencias comunicativas de niños y niñas han resultado altamente positivas, en tanto que ha permitido resolver simultáneamente dos dilemas básicos. El primero, superar la timidez y reforzar su desenvolvimiento por sí mismo con respecto al manejo del lenguaje. El segundo, en tanto que en esta edad el aprendizaje del lenguaje está todavía en su proceso inicial, la práctica de los juegos verbales a partir de su carácter lúdico, ha facilitado el desarrollo de su pensamiento, de su expresión oral, de su comprensión y el consiguiente incremento, acaso acelerado, de su vocabulario. 
Para este efecto, durante el proceso de desarrollo de la investigación se ha utilizado un conjunto de materiales didácticos especializados y elaborados bajo el principio de que dichos materiales de aprendizaje deben contextualizarse a la realidad directa e inmediata de los niños, esto con el objeto de facilitar fluida y progresivamente, así como sistemática y organizada, su capacidad de comunicación con los demás.

Las fuentes o referencias teóricas que se ha tomado en cuenta como soporte del presente estudio han sido, entre otras, la de Sigcha (2010) quien elaboró y aplicó un manual de ejercicios para desarrollar el lenguaje oral de los niños y niñas de 5 a 6 años de edad en una escuela mexicana. Como parte de sus resultados se recomienda: "hacer 18 intervenciones en el contexto lingüístico y extralingüístico del intercambio verbal, del tema de conversación, las actitudes y motivaciones de los participantes, al igual que las informaciones sobre la organización formal de los enunciados y las palabras que lo componen efectuar" (p. 67). Al respecto, sin embargo, es necesario hacerse la siguiente pregunta: ies suficiente las 18 intervenciones de intercambio verbal para decir que los niños o niñas han llegado a desarrollar sus habilidades comunicativas? Nosotros creemos que no, en tanto que dicho aprendizaje implica un proceso continuo y permanente que cubre todo el ciclo de crecimiento psicofísico del niño. La aplicación de las experiencias didácticas de comunicación deben tener también carácter permanente, es decir debe cubrir todo el proceso de desarrollo Inicial y Básica Regular de la Educación.

Otro referente teórico, que ha merecido ser tomado en cuenta, es la de Oña y Muñoz (2009). Dichos autores también han trabajado con niños y niñas de 5 a 6 años de edad, pero dando énfasis en la práctica del diseño de los juegos didácticos para conseguir el perfeccionamiento de las posibilidades comunicativas de los niños pero, he ahí la diferencia, "a partir de gestos simbólicos representacionales y su significatividad" (p. 19), es decir a partir de las estrategias de comunicación no verbal. Hecho que implica aceptar, que la construcción de la comunicación verbal se constituye en una segunda instancia dentro del proceso de aprendizaje del lenguaje. De ahí que podemos concluir afirmando que toda propuesta didáctica de aprendizaje de la comunicación verbal debe ser simultánea a los gestos, a la expresión y a la comprensión de significados.

Por lo demás, no abundan en el país estudios específicos sobre el tema de los juegos verbales como fuentes didácticas de aprendizaje de la comunicación; sin embargo, se ha podido ubicar algunos temas conexos, como sobre la comunicación oral (uso oral del lenguaje), el desarrollo del lenguaje, los juegos orales, etc. De estos trabajos 
resulta útil mencionar el estudio realizado por Villalba (2011) quien ha desarrollado investigación en nivel cuasi experimental (con un grupo experimental y otro de control). De sus resultados se resumen que "los juegos verbales como estrategias desarrollan la capacidad de comunicación oral, la articulación en la pronunciación y la coherencia de las palabras, el desarrollo de la audición, de la voz, de la entonación y gestos, la confianza y la seguridad al expresarse espontáneamente" (p. 79).

Siendo así, sobre la base de estas u otras propuestas teóricas, las mismas que se han ampliado en el marco teórico del texto original y las fuentes que respaldan la consistencia teórica de las variables de estudio, se ha formalizada la hipótesis en los términos siguientes:

\section{Hipótesis general}

La aplicación sistemática de los juegos lingüísticos tiene relación significativa y fuerte con el desarrollo de las habilidades comunicativas de los niños y niñas de 5 años de edad de Educación Inicial nº 652 de Supe-Barranca, en 2014.

\section{Hipótesis específicas}

HE1. El uso de las técnicas de comprensión influye en el desarrollo de las habilidades básicas de escuchar de los niños y niñas de 5 años de edad de Educación Inicial Nº 652 de Supe-Barranca, en 2014.

HE2. El uso de las técnicas expresivas influye en el desarrollo de las habilidades de hablar, de leer y escribir de los niños y niñas de 5 años de edad de Educación Inicial Nº 652 de Supe-Barranca, en 2014.

\section{Métodos y materiales}

El enfoque adoptado para la recopilación y tratamiento de las fuentes de información necesarias para la ejecución de la investigación han sido de carácter cualitativo; el tipo de estudio, descriptivo correlacional; y el diseño no experimental y de carácter transaccional; la población y la muestra de estudio, 60 niños y niñas seleccionados intencionalmente, siguiendo "el procedimiento no probabilístico" sustentado por Hernández et al (2015, p. 189), en tanto que se trabaja con una población pequeña.

El procedimiento metodológico seguido ha sido el de carácter lúdico, de entretenimiento y orientado siempre al desarrollo creativo del lenguaje. Con este propósito, dentro de 
las técnicas de comprensión y manejo de la capacidad comunicativa, se ha organizado progresivamente un conjunto de ejercicios verbales como el uso de trabalenguas, de las frases con rima, la repetición de canciones y presentación de cuentos breves de fácil asimilación o retención de su contenido. Como resultado, se ha conseguido desarrollar las capacidades de hablar, escuchar, leer y escribir de los niños y niñas que participaron en dicha experiencia.

Los materiales utilizados estuvieron concentrados en relación al uso diversificado de los ejercicios prácticos, pero en tanto contextualizados a la situación particular de los niños como, por ejemplo, el uso de papelotes, pizarra acrílica, guías de actividades pormenorizadas, según las exigencias previstas en las dimensiones e indicadores de estudio respectivos. Para este efecto, los instrumentos utilizados fueron validados previamente por juicio de expertos.

De igual manera, en lo que respecta a las técnicas expresivas, se organizaron actividades relacionadas a los siguientes ejercicios de aprendizaje: juego de roles, de dramatizaciones, de ejercicios bucales, de fonación (diferenciación del tono 0 acentuación), juegos expresivos (diferenciación de la melodía del habla), ejercicio buco-facial con el objeto que los niños y niñas se habitúen en la correcta pronunciación del sonido de las palabras; hechos que terminaron finalmente con la incrementación del léxico personal de los mismos.

Y para registrar dichos ejercicios se dispuso básicamente de una lista de cotejo, en la que se iba registrando el resultado de 40 indicadores previstos en la guía de actividades de aprendizaje, cuyos datos después de haberse aplicado en el semestre académico comprendido, entre abril a julio, arrojaron resultados teniendo en cuenta el siguiente rango previsto: inicio, en proceso y logro previsto. De ahí que a manera de resumen, se puede concluir afirmando que, la mayoría de los niños y niñas se ubicaron en nivel de proceso; en menor porcentaje, en inicio; y en el logro previsto, una cantidad casi insignificante.

\section{Resultados}

\subsection{Análisis descriptivo de las variables}

El primer paso consistió en medir la frecuencia de la variable independiente: los Juegos Lingüísticos" de los niños y niñas de 5 años de edad de la Institución Educativa de Educación Inicial No 652 de Supe-Barranca. De sus resultados se obtuvo la respuesta 
de que los niños y niñas de 5 años de edad estudiados, "el 65\% están en proceso; el $20 \%$ se encuentran en inicio; y $15 \%$ alcanzaron el logro previsto.

Además, en lo que respecta a dicha variable, se procedió a medir la frecuencia de sus respectivas dimensiones. La primera correspondió a la dimensión de "Técnicas comprensivas". De sus resultados, se obtuvo la respuesta que "el $68.3 \%$ se encuentran en proceso; el $21.7 \%$ en inicio; y $10 \%$ alcanzaron el logro previsto" (p. 57). La segunda medición correspondió a la dimensión "Técnicas expresivas". De sus resultados, se obtuvo la respuesta que "el $50 \%$ están en proceso; el $45.0 \%$ en inicio; y $5.0 \%$ en logro previsto" (p. 57).

El segundo paso consistió en medir la frecuencia de la variable dependiente: las "Habilidades comunicativas" de los niños y niñas de 5 años de edad de la Institución Educativa de Educación Inicial No 652 de Supe-Barranca. De sus resultados se colige que, con respecto a al desarrollo de las habilidades comunicativas, "el 53.3\% están en proceso; el $41.7 \%$ se encuentran en inicio; y el $0.5 \%$ en el logro previsto" (p. 58).

Dentro de esta variable se procedió también a medir la frecuencia de sus respectivas dimensiones. La primera correspondió a la "dimensión escuchar". De sus resultados se deduce que: el "51 (85\%) se encuentran en proceso de escuchar; 9 (15\%) se encuentran en la etapa de inicio; y ninguno (0\%) en el logro previsto" (p. 59); con los que queda demostrado que la habilidad de escuchar se convierte en la mejor destreza lograda por la población estudiantil y de la dimensión de "leer", se resume que el "52 (86.7\%) se encuentran en proceso de narrar con claridad los acontecimientos e ideas; $11(11.7 \%)$ se encuentran en inicio; y solo un niño o niña (1.7\%) se encuentra en el nivel óptimo de logro previsto" (p. 60). Asimismo en la dimensión de "escribir", , se deduce que "32 de niños o niñas (53.3\%) se encuentran en proceso de leer; 16 (26.7\%) en la etapa de inicio de leer; y 12 niños o niñas (20.0\%) se encuentra en el nivel óptimo del logro previsto" (p. 61). 


\subsection{Resultados inferenciales}

\subsubsection{Prueba de normalidad}

La Tabla 1 que se inserta, presenta los resultados de normalidad de KolmogorovSmirnov (K-S), con respecto a las variables y las dimensiones. Se observa que el valor de significancia asintótica (P) es mayor al nivel de significancia establecida ( $p$ $<.05)$, en el ajuste de la variable habilidades comunicativas y de las dimensiones: técnicas expresivas y técnicas de leer; por lo tanto, no hay diferencia significativa con la distribución normal; es decir, la configuración de la variable y dimensiones tienen distribución normal y por lo tanto se puede efectuar su comparación. La otra variable, correspondiente a los juegos lingüísticos, así como a sus respectivas dimensiones, como las técnicas de comprensión, de escuchar, de hablar y de escribir, no se aproximan a la distribución normal; por lo tanto, dichas correlaciones se realizaron con la prueba estadística no paramétrica: Coeficiente de Correlación de Spearman.

Tabla 1

Resultados de la prueba de normalidad Kolmogorov-Smirnov para las variables y dimensiones de estudio.

\begin{tabular}{lcc}
\hline Variables/Dimensiones & K-S & P \\
\hline Juegos Lingüísticos & 1.773 & .004 \\
Técnicas Comprensivas & 2.035 & .001 \\
Técnicas expresivas & .878 & .423 \\
Habilidades comunicativas & 1.223 & .101 \\
Escuchar & 1.884 & .002 \\
Hablar & 2.329 & .000 \\
Leer & 1.291 & .071 \\
Escribir & 1.781 & .004 \\
\hline
\end{tabular}

\section{Nivel de Significancia}

** Significativo $(p<.05)$

${ }^{\star \star \star}$ Muy significativo $(p<.01)$ 


\subsubsection{Juegos lingüísticos y habilidades comunicativas}

\section{Hipótesis general}

$H_{0}$ : No existe relación significativa entre los juegos lingüísticos y las habilidades comunicativas en los niños y niñas de 5 años de la Institución Educativa Inicial № 652 de Supe-Barranca, en 2014.

$\mathrm{H}_{1}$ : Existe relación significativa entre juegos lingüísticos y habilidades comunicativas en los niños y niñas de 5 años de edad de la Institución Educativa Inicial No 652 de Supe-Barranca, en 2014.

Tabla 2

Correlación de Spearman entre los juegos lingüísticos y habilidades comunicativas

\begin{tabular}{llccc}
\hline & & \multicolumn{1}{c}{$\begin{array}{c}\text { Juegos } \\
\text { Lingüísticos }\end{array}$} & $\begin{array}{c}\text { Habilidades } \\
\text { Comunicativas }\end{array}$ \\
\hline & Juegos & Coeficiente de correlación & 1,000 & $.986^{* *}$ \\
Rho de & Lingüísticos & Sig. (bilateral) &. & .000 \\
Spearman & $\mathrm{N}$ & 60 & 60 \\
& & Coeficiente de correlación & $986^{\star *}$ & 1,000 \\
& Habilidades & Sig. (bilateral) & .000 &. \\
& Comunicativas & $\mathrm{N}$ & 60 & 60 \\
\hline
\end{tabular}

**. La correlación es significativa al nivel 0,01 (bilateral).

Como se observa en la Tabla 2, podemos afirmar que existe una relación muy fuerte y perfecta entre los juegos lingüísticos y las habilidades comunicativas de los niños y niñas de 5 años de edad de la Institución Educativa Inicial No 652 de Supe-Barranca, en 2013. (sig. bilateral $=0.000<0.05$; Rho $=0.986$ ). Por lo tanto, rechazamos la hipótesis nula y aceptamos la hipótesis alterna.

\subsection{3 habilidades comunicativas y técnicas comprensivas}

$H_{0}$ : No existe relación significativa entre habilidades comunicativas y técnicas comprensivas de los niños y niñas de 5 años de edad de la Institución Educativa Inicial No 652 de Supe-Barranca, en 2014. 
$\mathrm{H}_{1}$ : Existe relación significativa entre habilidades comunicativas y técnicas comprensivas en los niños y niñas de 5 años de edad de la Institución Educativa Inicial No 652 del distrito de Supe provincia de Barranca 2014.

Tabla 3

Correlación de Spearman entre habilidades comunicativas y técnicas comprensivas

\begin{tabular}{lllcc}
\hline & & $\begin{array}{c}\text { Habilidades } \\
\text { comunicativas }\end{array}$ & $\begin{array}{c}\text { Técnicas } \\
\text { comprensivas }\end{array}$ \\
\hline & Habilidades & Coeficiente de correlación & 1,000 & $.945^{\star *}$ \\
Rho de & Comunicativas & Sig. (bilateral) &. & .000 \\
Spearman & & $\mathrm{N}$ & 60 & 60 \\
& Técnicas & Coeficiente de correlación & $.945^{\star *}$ & 1,000 \\
& Comprensivas & Sig. (bilateral) & .000 &. \\
& & $\mathrm{~N}$ & 60 & 60 \\
\hline
\end{tabular}

**. La correlación es significativa al nivel 0,01 (bilateral).

Como se observa en la Tabla 3 podemos afirmar que existe relación muy fuerte y perfecta entre habilidades comunicativas y técnicas comprensivas de los niños y niñas de 5 años de edad de la Institución Educativa Inicial № 652 de Supe-Barranca, en 2013. (sig. bilateral $=0.000<0.05$; Rho $=0.945$ ). Por lo tanto rechazamos la hipótesis nula y aceptamos la hipótesis alterna.

\subsection{4 habilidades comunicativas y técnicas expresivas}

\section{Hipótesis específica 2}

$\mathrm{H}_{0}$ : No existe relación significativa entre las habilidades comunicativas y las técnicas expresivas en los niños y niñas de 5 años de edad de la Institución Educativa Inicial No 652 de Supe-Barranca, en 2014.

$\mathrm{H}_{1}$ : Existe relación significante entre las habilidades comunicativas y las técnicas expresivas en los niños y niñas de 5 años de edad de la Institución Educativa Inicial № 652 de Supe-Barranca, en 2014. 
Tabla 4

Correlación de Spearman entre habilidades comunicativas y técnicas expresivas

\begin{tabular}{|c|c|c|c|c|}
\hline & & & $\begin{array}{c}\text { Habilidades } \\
\text { comunicativas }\end{array}$ & $\begin{array}{c}\text { Técnicas } \\
\text { expresivas }\end{array}$ \\
\hline \multirow{6}{*}{$\begin{array}{l}\text { Rho de } \\
\text { Spearman }\end{array}$} & \multirow{3}{*}{$\begin{array}{l}\text { Habilidades } \\
\text { Comunicativas }\end{array}$} & Coeficiente de correlación & 1,000 & $.974^{\star \star}$ \\
\hline & & Sig. (bilateral) & . & .000 \\
\hline & & $\mathrm{N}$ & 60 & 60 \\
\hline & \multirow{3}{*}{$\begin{array}{l}\text { Técnicas } \\
\text { expresivas }\end{array}$} & Coeficiente de correlación & $.974^{\star \star}$ & 1,000 \\
\hline & & Sig. (bilateral) & .000 & . \\
\hline & & $\mathrm{N}$ & 60 & 60 \\
\hline
\end{tabular}

De lo observado en la Tabla 4, se puede afirmar que existe relación muy fuerte y perfecta entre las habilidades comunicativas y las técnicas expresivas de los niños y niñas de 5 años de edad de la Institución Educativa Inicial № 652 de Supe-Barranca, en 2014. (sig. bilateral $=0.000<0.05 ;$ Rho $=0.974$ ). Por lo tanto, rechazamos la hipótesis nula y aceptamos la hipótesis alterna.

\section{DISCUSIÓN}

Los resultados alcanzados, tal como se ha demostrado en las respectivas tablas de análisis descriptivo de variables y así como en las de contrastación de hipótesis, han confirmado que la puesta en práctica de los juegos lingüísticos como estrategias didácticas para desarrollar las estrategias comunicativas en el nivel oral y escrita de los niños y niñas de cinco años de edad, han resultado altamente positivas y fuertes; ya que, según los niveles o categorías previstas en la tabla de evaluación correspondiente, el 53.3\% se han ubicado en el nivel "de proceso"; hecho que indica que el aprendizaje ha seguido un curso ascendente, sostenido y significativo; y el $41.7 \%$ en el nivel "de inicio", donde también se percibe que el aprendizaje ha sido ascendente, ya que la diferencia entre ambos niveles es muy cercana (7 \%); y solo $0.5 \%$ han alcanzado el nivel de "logro previsto", la misma que debe entenderse como un desarrollo de capacidad excepcional; aplicados solo en el primer semestre académico de 2014 (17 semanas). 
Sin embargo, es bueno recalcar respecto al margen de diferencia entre los dos primeros grupos: $53.3 \%$ el primero y $41.7 \%$ el segundo. Ellas indican que son escasamente el 7\% de diferencia. La validez de las hipótesis planteadas, al haber verificado que la aplicación de los juegos lingüísticos como estrategia didáctica para desarrollar las habilidades comunicativas de los niños y niñas de cinco años de edad, han sido altamente positivas y fuertes; ya que los resultados aludidos no destacan justamente por su diferencia sino, más bien, por su proximidad. De ahí que desde el punto de vista general y, siguiendo a Piaget (1986) como se citó en Nevado (2008), podemos afirmar que el aprendizaje del lenguaje de los niños debe concordarse a la actividad lúdica de los mismos, es decir debe formar parte de los "juegos de ejercicio" (p. 4). Este postulado, como se habrá podido deducir del parágrafo precedente, es pues acertado, ya que pondera el principio de que la enseñanza aprendizaje del niño debe asociarse a lo que más le atrae por su edad, esto es, el juego. Propuesta que ha sido asumida y verificada en la ejecución del trabajo que motiva el presente. Otro estudio que merece rescatarse, son los resultados alcanzados por uno de los más destacados teóricos de la psicología del desarrollo humano, Vygotsky quien sostuvo la teoría de que ya en los primeros años del niño se inicia el proceso de convergencia entre el lenguaje y el pensamiento, y por lo tanto la práctica de aquél está asociado al desarrollo de su inteligencia (pensamiento). Por esta razón la función de esas dos dualidades, estrechamente ligadas con son el "educador y el aprendiz", debe encaminarse en la misma dirección; ya que el niño "aprende a hablar hablando, esto supone saber usar el lenguaje más que conocer las reglas que la rigen" (p. 93). Hecho que pondera el principio de que la elección de las estrategias didácticas para impulsar el manejo del lenguaje debe iniciarse incluso desde los primeros años en que los niños y niñas empiezan a articular las palabras.

Pero el punto de vista que más se aproxima a los resultados alcanzados en la presente investigación, es lo sustentado por Sánchez et al (1996), citado por Roca (2013), quienes manifestaron que, en el desarrollo de la capacidad comunicativa, "el juego es una actividad que favorece el desarrollo del lenguaje. Los niños/as, mientras juegan, hablan constantemente [...] Cuando el niño/a comienza a hablar le gusta jugar con el lenguaje, deforma los sonidos o las palabras, habla con los labios juntos o con la boca abierta, dice palabras al revés" (p.15).

En efecto, el proceso de enseñanza aprendizaje del lenguaje debe ajustarse pues a las facultades naturales que posee el niño, es decir al juego, justamente por su condición de niño. Propuesta que debe tenerse en cuenta, como se ha hecho en el presente estudio, como uno de los factores influyentes en el proceso de enseñanza 
aprendizaje del lenguaje, pero en tanto se la asocie también a otros factores de mayor peso conceptual.

Otra propuesta que se asemeja a lo ejecutado en el presente estudio, es la de Villalba (2011) quién, según se desprende de los resultados insertados en su estudio, ha manejado mayor número de indicadores en comparación a otros revisados hasta aquí. La categoría o variable causal que la ha servido de base es "Los juegos verbales", la misma que le ha permitido desarrollar los siguientes indicadores: "la comunicación oral, la articulación en la pronunciación y coherencia de las palabras, el desarrollo de la audición, de la voz, de la entonación y gestos, la confianza y seguridad al expresarse espontáneamente" (p. 79).

En nuestro estudio se ha manejado y puesto en práctica también un buen número de indicadores lingüísticos y, acaso de mayor consistencia lúdica como, por ejemplo: el "ritmo, pulso, acento, dramatización, ejercicios bucólico faciales y ejercicios de fonación" (p. 24), y en tanto relacionados a las actividades de entretenimiento 0 juego, con el objeto de facilitar el desarrollo de las habilidades comunicativas de los niños y niñas, ya sea desde el punto de vista oral así como el escrito. De ahí que podemos afirmar, sin temor a equívoco, que la novedad y originalidad de los resultados alcanzados en el presente trabajo, se ha sustentado en torno a los indicadores que a continuación se explicitan: con el "ritmo", se ha inducido en los niños y niñas el sentido de la cadencia y compás en la asociación de los sonidos; con el "pulso", el movimiento y distribución armónica del sonido de las palabras; con el "acento", la pronunciación adecuada de los fonemas; con la "dramatización", la representación creativa de los contenidos percibidos por ellos mismos; con los "ejercicios bucólico-faciales", la correcta articulación y pronunciación de las sílabas; y con los "ejercicios de fonación", la distinción o diferenciación de los fonemas para una correcta organización de las palabras.

Larco (2007) también ha aplicado nuevas estrategias didácticas en su estudio, pero enfocados específicamente al desarrollo de la habilidad comunicativas de los niños bajo la denominación de: "Programa de desarrollo de la inteligencia lingüística para favorecer procesos mentales a través de la lectura y escritura" (p. 67). Aquí, lo que se ha pretendido medir, según se puede deducir de sus objetivos insertados, son los "procesos mentales" básicos, es decir la capacidad de comprensión lectora. Hecho que resulta pues un proceso mayor y más complejo, que la pura ejercitación del niño o niña en el manejo de la pronunciación u organización lexical. Siendo así, en 
nuestro trabajo dicho proceso mental ha estado asociado también al procedimiento de conocimiento y manejos del lenguaje pero, he aquí la diferencia, a partir de la actividad lúdica. Además, otro estudio que se asemeja a lo realizado en la presente investigación, es el de Gamarra y Saldaña (2012), quienes concentraron su estudio sobre la base de la aplicación de los "roles" de los niños y niñas de 5 años de edad. Para este efecto, utilizaron como variable de estudio, "Los roles del niño". A diferencia del estudio realizado en nuestro caso, ellos se concentraron más bien en la discriminación auditiva de los "sonidos onomatopéyicos de su entorno", así como en el desarrollo de las habilidades fonológicas siguientes: "silabeo de palabras: directas, inversas, trabadas, simples y complejas, sean estas largas o cortas, así como seguir la secuencia fonética de frase y oración [... ] desarrollo de: rimas, trabalenguas, poesías, canciones, como las actividades literarias que despiertan interés y atención y concentración, especialmente en los más inquietos" (p.23). Sin embargo, de los indicadores aludidos, habría la necesidad de precisar el alcance conceptual de la categoría "actividades literarias". A nuestro parecer, así como está insertada en el texto, dicho tratamiento resulta muy ambiguo y alude a un conjunto ilimitado de acciones que puedan adoptarse para dinamizar el manejo del lenguaje. En el caso nuestro, dicho tema ha sido asumido en el aspecto concreto de la ejercitación fonética y escritural breve e inicial del lenguaje. Por eso tal vez esta sea uno de los retos que pueda derivarse de nuestro estudio, es decir la necesidad de insistir en el manejo de las categorías literarias no solo para coadyuvar en el uso de estrategias sobre el manejo oral y escrito del lenguaje, sino también para generar acaso la opción de creación estética en nuestros alumnos.

En conclusión, sobre la base de las consideraciones aludidas, podemos reafirmar sin temor a equívoco que, la aplicación de los juegos lingüísticos como estrategias didácticas para desarrollar la capacidad de manejo del lenguaje de los niños y niñas de cinco años de edad, ya sea desde el punto de vista oral así como del escrito, han sido altamente positivas y fuertes; cuyas pruebas se sustentan en las tablas de descripción de variables así como en las de contrastación de hipótesis, ya explicitadas en los rubros precedentes. 


\section{REFERENCIAS}

Cruz, J. (2013). Influencia del programa IBM Kidmart en el desarrollo de las habilidades comunicativas. Lima: Universidad Privada César Vallejo.

Larco, M. (2007). Programa de desarrollo de inteligencia lingüística para favorecer procesos mentales a través de la lectura y escritura en niños de primer año de educación básica, en Jardines de infantes fiscales del sector Inca de Quito. Universidad Tecnológica Equinoccial. Ecuador. Recuperado de http:// repositorio.ute.edu.ec/handle/123456789/7661

Nevado, Ch. (2008). El componente lúdico en las clases de ELE. Marco. Revista de didáctica ELE. N 7: 1-14.

Oña, S. y Muñoz, M. (2009). Perfeccionamiento de la comunicación en niñas/os de cinco a seis años que asisten al círculo infantil basados en juegos didácticos. Unidad Académica de Ciencias Administrativas y Humanísticas. UTC. Latacunga. Recuperado de http://repositorio.utc.edu.ec/handle/27000/323.

Piaget, J. (1968/1976). El lenguaje y el pensamiento en el niño. Estudio sobre la lógica del niño (I). Buenos Aires: Editorial Guadalupe.

Roca, E. (2013). La estimulación del lenguaje en educación infantil: un programa de intervención en el segundo ciclo de educación infantil. Escuela Universitaria de Magisterio. Universidad de Valladolid. Recuperado de https://uvadoc.uva. es/bitstream/10324/3226/1/TFG-B.241.pdf

Sigcha, M. (2010). Elaboración y aplicación de un manual de ejercicios para desarrollar el lenguaje oral de niños y niñas de 5 a 6 años. Escuela de práctica docente "Agustín Albán" del cantón Pujilí Barrio Guápulo, periodo escolar 20092010. Unidad Académica de Ciencias Administrativas y Humanísticas. UTC. Latacunga. Recuperado de http://repositorio.utc.edu.ec/handle/27000/279.

Vigotsky, L. (1998). Pensamiento y lenguaje. Buenos Aires: Fausto.

Villalba, A. (2011). Aplicación de los juegos verbales como estrategia en el desarrollo de la comunicación oral a niños de primer grado de la I.E." PERÚ BIRF de Juliaca-Puno 